\title{
A propos des variations de la composition minérale des laits durant l'été 1976
}

INFLUENCE DE LA SECHERESSE (1)

\author{
par \\ F. M. LUQUET, H. MAHIEU et L. MOUILLET
}

La sécheresse durant l'été 1976, a posé de nombreux problèmes aux producteurs et aux transformateurs de lait. L'un d'eux tenait à la conservation des laits dont les équilibres salins semblaient fortement perturbés. Un fait similaire avait déjà été observé les années précédentes dans les enquêtes réalisées.

"A propos de la teneur des laits individuels et de mélange en matières minérales et urée sur des laits de producteurs (1973) et sur des laits de grand mélange (1974-1975) » (1).

Afin de trouver un remède à de nombreux accidents pouvant avoir lieu depuis le stockage à la ferme jusqu'à la fabrication en laiterie, il a été demandé de faire un test rapide sur la composition des laits en divers éléments, et en particulier en éléments minéraux.

$$
\text { * * }
$$

\section{ECHANTILLONNAGE ET METHODES}

\section{Echantillonnage}

Une première série d'échantillons a été sollicitée au début du mois de septembre 1976 ; la sécheresse durait depuis plus de 2 mois : dix-neuf usines ont répondu à cet appel (échantillon moyen concernant la période du $1^{\text {er }}$ au 15 septembre 1976).

(1) Travaux effectués dans le cadre du Labcodral : E.N.S.I.A., 105, rue de l'Université - 59509 Douai. 
Un deuxième appel a été effectué à la fin du mois d'octobre 1976 ; la situation était redevenue à peu près normale depuis 1 mois : vingt usines y ont répondu (échantillon moyen concernant la période du 20 au 30 octobre 1976).

L'échantillonnage est présenté dans le tableau 1 et situé sur la figure 1 ; avec mention de la comparaison avec les enquêtes précédentes (2).

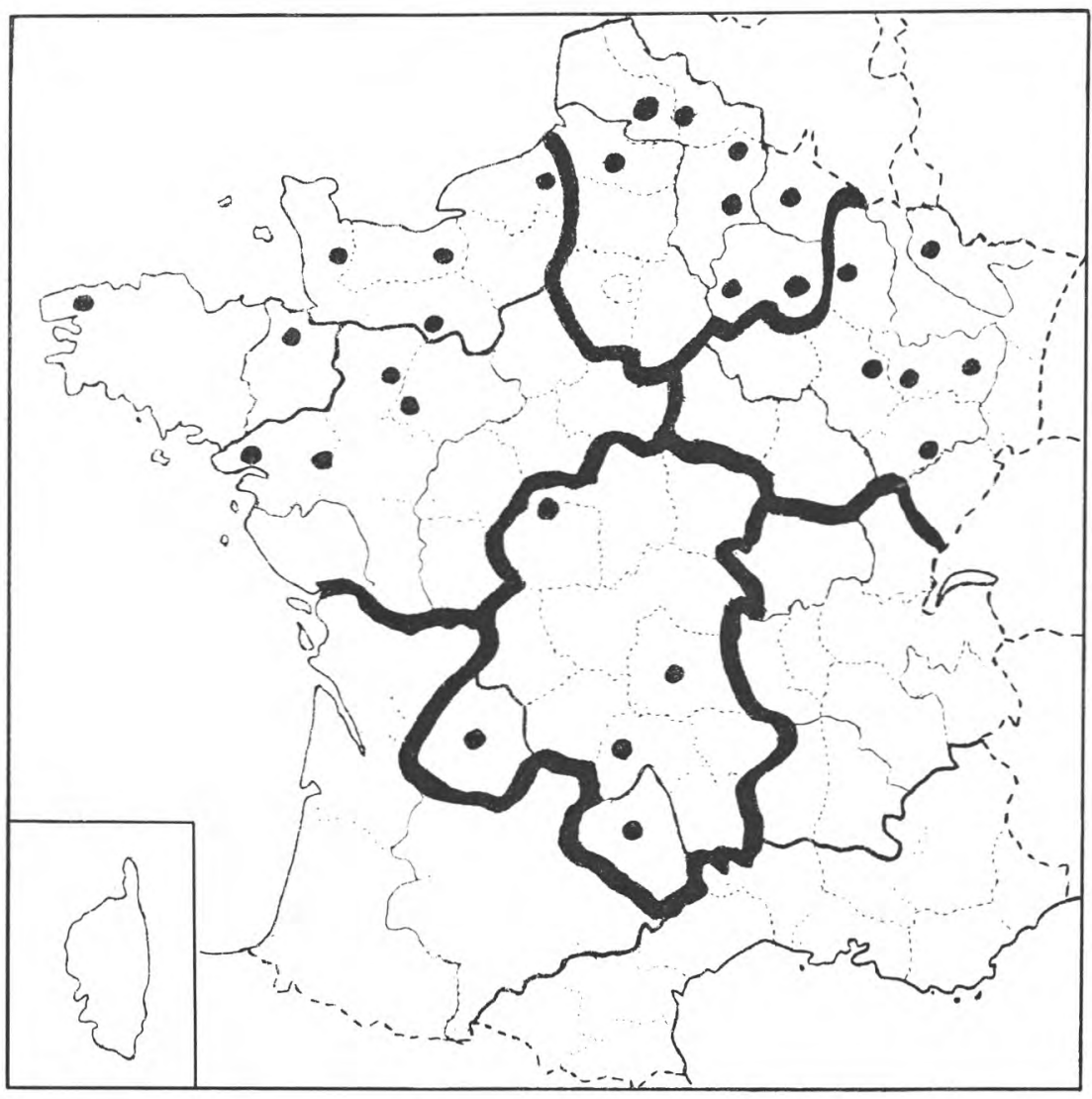

fig. 1

Echantillonnage (carte des zones)

(2) A propos de la teneur des laits individuels et de mélange en matières minérales et urée. Le Lait, cf. bibliographie, 3. 
TABLEAU 1

L'échantillonnage

\begin{tabular}{|c|c|c|c|c|c|}
\hline \multirow{2}{*}{ Régions } & \multirow{2}{*}{$\begin{array}{l}\text { Correspondances } \\
\text { enquêtes } \\
1973 \text { à } 1975\end{array}$} & \multicolumn{2}{|c|}{$\begin{array}{l}\text { Nombre } \\
\text { de } \\
\text { laiteries }\end{array}$} & \multicolumn{2}{|c|}{$\begin{array}{c}\text { Litrages } \\
\text { correspondants } \\
\text { (hl) }\end{array}$} \\
\hline & & $\begin{array}{l}\text { sept. } \\
1976\end{array}$ & $\begin{array}{l}\text { oct. } \\
1976\end{array}$ & $\begin{array}{l}\text { sept. } \\
1976\end{array}$ & $\begin{array}{l}\text { oct. } \\
1976\end{array}$ \\
\hline Est & Est & 4 & 6 & 2700 & 5030 \\
\hline Nord-Est & Nord-Est & 6 & 4 & 9130 & 3500 \\
\hline Grand-Ouest & $\begin{array}{l}\text { Normandie - Bretagne } \\
\text { Ouest }\end{array}$ & 5 & 8 & 15000 & 7900 \\
\hline Centre - Massif Central & $\begin{array}{l}\text { Centre - Massif Central } \\
+ \text { Dordogne }\end{array}$ & 4 & 2 & 3000 & 2000 \\
\hline France & & 19 & 20 & 29830 & 18430 \\
\hline
\end{tabular}

\section{Méthodes de dosage}

Les méthodes utilisées furent les mêmes que celles décrites lors de la publication des enquêtes précédentes (2).

Spectrophotométrie d'absorption atomique :

Calcium

Potassium

Magnésium

Sodium
Fer

Cuivre

Manganèse

Zinc

Colorimétrie : Phosphore.

\section{Précautions opératoires}

Il a été tenil compte dans cette enquête des leçons tirées des précédentes :

(2) A propos de la teneur des laits individuels et de mélange en matières minérales et urée. Le Lait, cf. bibliographie, 3. 
- il a été rempli autant de flacons de $250 \mathrm{~cm}^{3}$ par échantillon que de séries d'analyses à effectuer : soit quatre pour permettre des contre-analyses si nécessaire ;

- afin d'éviter les interférences analytiques, aucun conservateur n'a été utilisé ;

- n'ont été conservés que les échantillons parvenus rapidement et dans de bonnes conditions au laboratoire ;

- à l'arrivée au laboratoire les flacons ont été immédiatement congelés, pour n'être ressortis que lors des analyses correspondantes.

Afin de juguler le plus possible les facteurs d'erreur dans les résultats ; non seulement les analyses ont été effectuées dans le même laboratoire, mais encore par le même technicien. De ce fait une différence de 5 p. 100 entre deux analyses commence à présenter une signification, qui devient d'autant plus réelle à 10 p. 100 .

\section{Résultats octobre 1974}

Afin de pouvoir différencier ce qui est dû à la sécheresse de ce qui est dû à l'évolution de la composition minérale des laits entre 1974 et 1976, le tableau des résultats enregistrés au mois d'octobre 1974 a été établi en fonction de la régionalisation de l'enquête du mois d'octobre 1976 (tab. 2).

TABLEAU 2

Résultats octobre 1974

\begin{tabular}{|c|c|c|c|c|c|c|c|c|c|}
\hline & \multicolumn{5}{|c|}{ Exprimés en mg/l } & \multicolumn{4}{|c|}{ Exprimés en $\mu \mathrm{g} / 1$} \\
\hline & $\mathrm{Ca}$ & $P$ & K & $\mathrm{Mg}$ & $\mathrm{Na}$ & $\mathrm{Fe}$ & $\mathrm{Ca}$ & $\mathrm{Mn}$ & $\mathrm{Zn}$ \\
\hline Est & 1196 & 924 & * & 111 & 475 & 687 & 218 & 53 & 3970 \\
\hline Nord-Est & 1222 & 902 & * & 115 & 514 & 1054 & 205 & 70 & 3376 \\
\hline Grand-Ouest & 1257 & 965 & * & 116 & 495 & 610 & 204 & 58 & 3964 \\
\hline $\begin{array}{l}\text { Centre } \\
\text { Massif Central }\end{array}$ & 1208 & 912 & * & 112 & 476 & 447 & 295 & 54 & 3350 \\
\hline France & 1222 & 951 & * & 113 & 487 & 631 & 224 & 60 & 3830 \\
\hline
\end{tabular}




\section{RESULTATS}

L'ensemble des résultats est présenté dans les deux pages suivantes sous forme de tableaux :

Tab. 3 : Résultats septembre 1976.

Tab. 4 : Résultats octobre 1976.

Tab. 5: Influence de la sécheresse représentée par le rapport : Résultat septembre 1976

Résultat octobre 1976 exprimé en pourcentage.

Tab. 6: Evolution de la composition minérale des laits entre 1974 et 1976 représentée par le rapport :

Résultat octobre 1976

Résultat octobre 1974 exprimé en pourcentage.

Remarques : Symboles utilisés

- éléments plastiques (résultats exprimés en $\mathrm{mg} / \mathrm{l}$ )

$\mathrm{Ca}=$ Calcium

$\mathrm{P}=$ Phosphore

$\mathrm{K}=$ potassium

$\mathrm{Mg}=$ Magnésium

$\mathrm{Na}=$ Sodium

- oligoéléments

$\mathrm{Fe}=\mathrm{Fer}$

(résultats exprimés en $\mu \mathrm{g} / 1$ )

$\mathrm{Cu}=$ Cuivre

$\mathrm{Mn}=$ Manganèse

- signes :

$\mathrm{Zn}=\mathrm{Zinc}$

$=$ Les résultats sont identiques.

\# Les résultats sont peu différents.

* Le potassium n'a pu être analysé en octobre 1974.

- Numérateur inférieur au dénominateur de tant p. 100.

+ Numérateur supérieur au dénominateur de tant p. 100.

\section{COMMENTAIRES}

\section{Influence de la sécheresse sur la composition minérale des laits}

En comparant les moyennes arithmétiques des résultats des analyses effectuées en octobre 1976 à celles des analyses effectuées en septembre 1976, l'on peut observer que :

- les teneurs en Magnésium, Sodium et Zinc restent semblables ; 
TABLEAU 3

Résultats septembre 1976

\begin{tabular}{|c|c|c|c|c|c|c|c|c|c|}
\hline & \multicolumn{5}{|c|}{ Exprimés en mg/l } & \multicolumn{4}{|c|}{ Exprimés en $\mu \mathrm{g} / \mathrm{l}$} \\
\hline & $\mathrm{Ca}$ & $P$ & $\mathrm{~K}$ & $\mathrm{Mg}$ & $\mathrm{Na}$ & $\mathrm{Fe}$ & $\mathrm{Cu}$ & Mn & $\mathrm{Zn}$ \\
\hline Est & 1164 & 905 & 1337 & 104 & 456 & 737 & 142 & 53 & 4198 \\
\hline Nord-Est & 1182 & 898 & 1335 & 109 & 487 & 819 & 139 & 58 & 4292 \\
\hline Grand-Ouest & 1194 & 911 & 1323 & 106 & 444 & 805 & 129 & 64 & 4054 \\
\hline $\begin{array}{l}\text { Centre } \\
\text { Massif Central }\end{array}$ & 1119 & 855 & 1321 & 100 & 426 & 671 & 119 & 54 & 3493 \\
\hline France & 1174 & 898 & 1325 & 106 & 458 & 782 & 130 & 58 & 4089 \\
\hline
\end{tabular}

TABLEAU 4

Résultats octobre 1976

\begin{tabular}{|c|c|c|c|c|c|c|c|c|c|}
\hline & \multicolumn{5}{|c|}{ Exprimés en mg/l } & \multicolumn{4}{|c|}{ Exprimés en $\mu \mathrm{g} / \mathrm{l}$} \\
\hline & $\mathrm{Ca}$ & $\mathrm{P}$ & $\mathrm{K}$ & $\mathrm{Mg}$ & $\mathrm{Na}$ & $\mathrm{Fe}$ & $\mathrm{Cu}$ & Mn & $\mathrm{Zn}$ \\
\hline Est & 1205 & 937 & 1348 & 106 & 421 & 551 & 127 & 55 & 4153 \\
\hline Nord-Est & 1239 & 952 & 1371 & 106 & 419 & 615 & 124 & 55 & 4310 \\
\hline Grand-Ouest & 1213 & 931 & 1278 & 106 & 419 & 793 & 140 & 59 & 3825 \\
\hline $\begin{array}{l}\text { Centre } \\
\text { Massif Central }\end{array}$ & 1174 & 876 & 1379 & 103 & 405 & 681 & 123 & 53 & 4076 \\
\hline France & 1221 & 936 & 1337 & 106 & 414 & 663 & 133 & 55 & 4077 \\
\hline
\end{tabular}




\section{TABLEAU 5}

Influence de la sécheresse représentée par le rapport : résultat septembre 1976 résultat octobre 1976

\begin{tabular}{l|c|c|c|c|c|c|c|c|c}
\hline & $\mathrm{Ca}$ & $\mathrm{P}$ & $\mathrm{K}$ & $\mathrm{Mg}$ & $\mathrm{Na}$ & $\mathrm{Fe}$ & $\mathrm{Cu}$ & $\mathrm{Mn}$ & $\mathrm{Zn}$ \\
\hline Est & & & & & & & & & \\
& & & & \\
Nord-Est & $-4,5$ & $-3,5$ & H & $-1,9$ & $+9,2$ & $+33,7$ & $+11,8$ & $-3,8$ & $+1,1$ \\
Grand-Ouest & $-1,6$ & $-2,2$ & $+3,5$ & $=$ & $+6,0$ & $+1,5$ & $-7,9$ & $+8,5$ & $+6,0$ \\
Centre & & $-2,7$ & $+2,8$ & $+14,0$ & $+33,2$ & $+12,1$ & $+5,2$ & $\#$ \\
Massif Central & $-4,7$ & $-2,4$ & $-4,2$ & $-2,9$ & $+5,2$ & $-1,5$ & $-3,3$ & $+1,9$ & $-14,3$ \\
\hline France & $-3,8$ & $-4,0$ & $-0,9$ & $=$ & $+10,6$ & $+17,9$ & $-2,3$ & $+5,5$ & $\#$ \\
\hline
\end{tabular}

\section{TABLEAU 6}

Evolution de la composition minérale des laits entre 1974 et 1976 représentée résultat octobre 1976

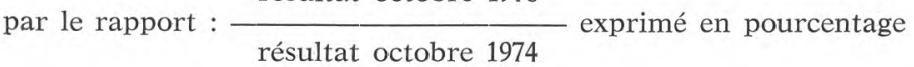

\begin{tabular}{l|c|c|c|c|c|c|c|c|c}
\hline & $\mathrm{Ca}$ & $\mathrm{P}$ & $\mathrm{K}$ & $\mathrm{Mg}$ & $\mathrm{Na}$ & $\mathrm{Fe}$ & $\mathrm{Cu}$ & $\mathrm{Mn}$ & $\mathrm{Zn}$ \\
\hline Est & $+0,8$ & $+1,4$ & $*$ & $-4,5$ & $-11,4$ & $-19,8$ & $-41,7$ & $+3,8$ & $+4,6$ \\
\hline Nord-Est & $+1,4$ & $+5,5$ & $*$ & $-7,8$ & $-18,5$ & $-41,7$ & $-39,5$ & $-21,4$ & $+27,7$ \\
Grand-Ouest & $-3,5$ & $-3,5$ & $*$ & $-8,6$ & $-15,4$ & $+30,0$ & $-31,4$ & $+1,7$ & $-3,5$ \\
Centre & & & & & & & & \\
Massif Central & $-2,8$ & $-3,9$ & $*$ & $-8,0$ & $-14,9$ & $+52,3$ & $-58,3$ & $-1,8$ & $+21,7$ \\
\hline France & $\#$ & $-1,6$ & $*$ & $-6,2$ & $-15,0$ & $+5,1$ & $-40,6$ & $-8,3$ & $+6,4$ \\
\hline
\end{tabular}


- les teneurs en Calcium et Phosphore marquent même une légère diminution ; sensiblement du même ordre dans les différentes régions de l'enquête 2 à 6 p. 100 ;

- les teneurs en Cuivre et Fer ne traduisent qu'une variation de la contamination du lait après la traite ;

- seules les variations des teneurs en Manganèse et surtout en Sodium semblent significatives.

Il avait déjà été constaté qu'en cas de stress (mammites par exemple) la mamelle, pour équilibrer sa pression osmotique réagissait en faisant appel à certains ions du plasma sanguin et en particulier au Sodium ; en contrepartie la teneur en certains ions diminuait : Calcium, Phosphore... Un phénomène du même ordre pourrait avoir eu lieu à l'occasion de la sécheresse de l'été 1976.

\section{Evolution de la composition minérale des laits entre 1974 et 1976}

En comparant les moyennes arithmétiques des résultats des analyses effectuées en octobre 1976, à celles des analyses effectuées en octobre 1974, l'on peut observer que :

- Les teneurs en Calcium et Phosphore sont identiques.

La teneur en Calcium qui avait baissé en 1975 s'est redressée. Le rapport Calcium/Phosphore se maintient proche de 1,31. Dans les régions, si les variations semblent plus importantes, elles sont toutefois semblables (cependant augmentation du Phosphore dans la région Nord-Est de 5 p. 100).

- La contamination du lait par le Cuivre après la traite a fortement diminué : - 40,6 p. 100.

Ceci est dû à l'élimination de ce métal dans les matériaux constitutifs des équipements de laiteries, de fermes et de circuits de ramassage. Le Massif Central a l'évolution la plus importante.

- La contamination du lait par le Fer après la traite a varié différemment selon les régions. Elle a fortement diminué dans le Nord-Est et l'Est de la France et particulièrement augmenté dans les autres régions.

- Les teneurs en Magnésium, Sodium et Manganèse ont assez sensiblement baissé.

Cette baisse concerne principalement la teneur en Sodium ; elle est identique dans toutes les régions de même que celle en Magnésium, mais à un moindre degré. Seule la région Nord-Est présente une baisse importante de la teneur des laits en Manganèse.

- La teneur en Zinc est en augmentation, principalement dans le Nord-Est et le Centre-Massif Central. 
Si l'on s'en réfère à la bibliographie concernant l'action des fortes chaleurs sur la composition du lait, de nombreuses études ont été effectuées principalement en France et aux Etats-Unis.

\section{Travaux français effectués à l'Ecole Nationale Vétérinaire d'Alfort}

L'action déprimante des fortes chaleurs sur la production a pour conséquence une diminution des ingesta et une augmentation de l'évaporation pulmonaire concomitante à une faible sécrétion de tyroxine durant ces périodes. La composition du lait change; on constate :

- une augmentation de l'azote non protéique ;

des acides palmitiques et stéariques ;

- une diminution des lipides totaux ;

de la matière sèche ;

de l'azote totale ;

du lactose ;

de l'acide oléique ;

des acides gras à courte chaîne $\left(\mathrm{C}_{6}\right.$ à $\left.\mathrm{C}_{10}\right)$.

\section{Etudes américaines}

Des chercheurs de l'Université du Missouri, opérant en chambre conditionnée, ont déterminé que la température de confort des vaches se situait entre $0^{\circ} \mathrm{C}$ et $16^{\circ} \mathrm{C}$. Au-dessus de $27^{\circ} \mathrm{C}$ pour les Holstein et de $29,5^{\circ} \mathrm{C}$ pour les Jersey et les Brown-Swiss des températures constantes provoquent une baisse de la production de lait et des teneurs en matières azotées et en lactose.

Entre $5^{\circ} \mathrm{C}$ et $27^{\circ} \mathrm{C}$, le taux butyreux diminue, en sens inverse de la température, il augmente pour des températures supérieures à $27^{\circ} \mathrm{C}$ et inférieures à $5^{\circ} \mathrm{C}$, en même temps que la quantité de lait diminue.

Johson et al. (1966) ont exposé des vaches à un environnement à $31^{\circ} \mathrm{C}$ et contraint celles-ci par l'intermédiaire d'une fistule de rumen à consommer autant d'aliment que des vaches témoins nourries ad libitum dans un environnement à $18^{\circ} \mathrm{C}$. Comparé avec les animaux nourris ad libitum à $31^{\circ} \mathrm{C}$, les animaux forcés à $31^{\circ} \mathrm{C}$ ont une production laitière et une température rectale légèrement supérieure. Comparé avec les résultats obtenus à $18^{\circ} \mathrm{C}$, les animaux poussés à $31^{\circ} \mathrm{C}$ à manger la même alimentation ont montré toutefois :

- une baisse de la production laitière ;

de la production laitière corrigée ;

de l'extrait sec;

de l'extrait sec dégraissé ;

de la matière grasse ; 
- une moindre efficience du rapport :

prise de T.D.N. (Total Digestif Nutrient)

production de lait corrigée/M.G.

Moody et al. (1967) concluent que l'effet stressant des hautes températures lors de ces différentes baisses, ne peut être qu'en partie expliqué par une baisse de la consommation d'aliments. Leurs vaches nourries ad libitum à $32^{\circ} \mathrm{C}$ consommaient de l'énergie en excès ; leur production de lait corrigée par rapport à la matière grasse baissait de 26,9 p. 100 et 37,8 p. 100 à la fin de 1 à 2 semaines d'exposition, alors que la consommation d'énergie digestible avait décru de 14,6 p. 100 et 18,3 p. 100.

Johson et al. (1967) ont montré que l'effet dépressif des hautes températures sur la prise d'aliments et la production laitière n'est pas un effet à court terme. La dépression est maximale après 2 semaines ; il y a un léger redressement vers les 3-5 semaines : les animaux semblent en équilibre, mais sont moins productifs qu'à $18^{\circ} \mathrm{C}$.

Maust, Mc Dowell et Hoover (1972) ont précisé que cette dépression est plus marquée en milieu de lactation (100-180 j).

Ces changements dans la composition du lait, observés dans le cas de témpératures ambiantes élevées peuvent transformer les propriétés de stockage et de traitement du lait.

Moody et al. (1971) ont étudié ces variations en comparant pendant 2 semaines le lait de vaches vivant dans un environnement naturel $\left(15-24^{\circ} \mathrm{C}\right)$ et de vaches maintenues dans une température ambiante de $32^{\circ} \mathrm{C}$ (humidité relative 60 p. 100). Les animaux exposés à une forte chaleur présentent :

- une baisse du poids corporel ;

de la production laitière ;

de la quantité de matière grasse sécrétée;

- dans cette matière grasse, la production des acides gras change il y a une diminution des acides gras à courte chaîne, des acides laurique myristique, muristoléique, pentadocénoïque, palmitoléique, oléique et linoléique ; les proportions d'acides palmitiques et stéariques sont en hausse.

On explique ces changements en supposant une réduction dans la fourniture d'acides gras provenant des fermentations du rumen et une augmentation des acides gras originaires des matières grasses corporelles mobilisées.

De nombreux travaux ont montré qu'aux fortes températures, il y a réduction de la concentration des acides gras volatils dans 
le liquide du rumen. Cette réduction n'est pas due qu'à la réduction de la prise d'aliment, elle n'est pas une conséquence directe d'une augmentation de la température du rumen, ni de l'augmentation de la consommation d'eau.

Hartz et al. (1971) ont montré une réduction des fermentations et des mouvements dans le rumen.

Aux fortes températures, la balance hydrique des animaux est perturbée ; Mc Dowell et al. (1969) ont constaté sur des vaches Holstein en lactation, exposées à une température de $32^{\circ} \mathrm{C}$ pendant 2 semaines :

- une réduction de leur consommation d'aliments et de leur production laitière ;

- une augmentation de leur consommation d'eau de 28 p. 100 allant de pair avec une diminution de l'eau dans les fécès de 33 p. 100 et une augmentation de l'eau dans le sang et les urines, et du volume des urines.

\section{EN GUISE DE CONCLUSION}

Au cours de cette enquête, il a pu être constaté que les variations dues à l'année étaient supérieures à celles dues à la sécheresse. Seules les variations de la teneur du lait en Sodium et à un moindre degré des teneurs en Calcium et en Phosphore pourraient être imputables à cette calamité.

On a pu observer entre les mois correspondants des années 1974 et 1976 :

- une stabilisation à un niveau assez bas des teneurs en Calcium et en Phosphore ;

- une chute des teneurs en Magnésium et en Sodium (même pas entièrement compensée pour le Sodium durant la sécheresse) ; le Cuivre ;

- une diminution des contaminations par le Fer et surtout par

- un maintien dans la conformité des teneurs en Manganèse et en Zinc ; mais avec une tendance à la diminution pour le Manganèse et à l'augmentation pour le Zinc.

Les teneurs du lait en éléments minéraux ont une tendance à la baisse, excepté peut-être pour celle en Zinc. Les efforts faits dans certaines régions pour mieux nourrir les animaux pourraient constituer une explication au léger redressement des teneurs en Calcium, Phosphore et Zinc, bien qu'il faille rester prudent en ce domaine. 


\section{Bibliographie}

1. Roux (M.), Luduet (F. M.) et Casalis (J.) (1974). - Sur lá teneur en sels minéraux du lait de vache des régions du Nord et du Sud-Ouest de la France. Ann. Fals. exp. chim., rov.-déc., 67, n 723-724, 577-583.

2. Mouillet (L.), Luguet (F. M.) et Casalis (J.) (1975). - Contribution à l'étude des variations de la teneur en sels minéraux du lait de vache dans différentes régions françaises. Le Lait, tome LV, n 549-550, nov.-déc., 683-694.

3. Mahieu (H.), Luquet (F. M.) et Mouillet (L.) (1976). - A propos de la teneur des laits individuels et de mélange en matières minérales et urée. Le Lait, tome LVI, $\mathrm{n}^{\circ}$ 559-560, nov.-déc., 657-697.

4. Mahieu (H.), Luquet (F. M.) et Mouillet (L.) (1977). - A propos de la teneur des laits individuels et de mélange en matières minérales et urée. Le Lait, tome LVII, n 561-562, janv.-fév., 55-112.

5. Mahieu (H.), Luouet (F. M.) et Mouillet (L.) (1977). - A propos de la teneur des laits individuels et de mélange en matières minérales et urée. Le Lait, tome LVII, no 563-564, mars-avril, 184-214.

6. Thompson (G. E.). - Review of the progress of dairy science climatic physiology of cattle. J. of Dairy Research, vol. 40, 441-473. 